\title{
Optimal One-Shot Scheduling for MIMO Networks
}

\author{
Douglas M. Blough, Giovanni Resta, Paolo Santi, Ramya Srinivasan, and Luis Miguel Cortés-Peña* \\ ${ }^{*}$ D. Blough, R. Srinivasan, and L.M. Cortés-Peña are with the Georgia Institute of Technology, Atlanta, Georgia, USA. \\ G. Resta and P. Santi are with the Istituto di Informatica e Telematica del CNR, Pisa, Italy.
}

\begin{abstract}
A MIMO network is a wireless network made up of individual MIMO links. The problem we consider is to maximize throughput in a multihop MIMO network with interference suppression. Our problem formulation accounts for variable rates on the MIMO links, which depend on the channel conditions of the link, and the manner in which the diversity-multiplexing trade-off is handled. We present an ILP formulation of the MIMO one-shot scheduling problem with variable rates, which is the first exact formulation of a MIMO network optimization problem that accounts for full interference suppression capabilities of MIMO links. We use CPLEX to evaluate the optimal solution based on the ILP formulation for wireless networks with up to 32 concurrently transmitting links. We also modify a heuristic algorithm from a related MIMO scheduling problem to work in our problem setting. Results show that the heuristic can scale to networks with 80 or more concurrent links, but is 10$20 \%$ from optimal in terms of throughput. We show that the heuristic scheduler is not able to fully exploit the diversitymultiplexing-interference suppression tradeoff, which is inherent in the problem. This shows that there is substantial room for developing improved scheduling algorithms for MIMO networks and provides some insight into promising directions to explore.
\end{abstract}

\section{INTRODUCTION}

Deployments of all-wireless networks are increasing rapidly due to the emergence of wireless mesh networks and WiMax. If these networks are to compete with wired networks in terms of performance, techniques to maximize throughput in all-wireless networks must be developed. One of the most promising approaches to throughput improvement in wireless networks is the use of multiple-input multiple-output, or MIMO, technology. Applying MIMO on individual links can provide an immediate throughput boost to these networks. However, optimizing the use of MIMO resources across the entire network has the potential to improve throughput by an even greater amount. For example, our prior work has demonstrated that the total number of concurrent streams that can be supported across a network can be doubled if networkwide optimization techniques are employed, as compared to link-by-link optimization [17].

One of the key factors that distinguish the MIMO network optimization problem from one in which link-by-link optimization is performed is the capability of MIMO links to perform interference suppression. Eliminating interference allows greater spatial reuse, which increases the overall capacity of a wireless network. However, the use of MIMO resources for interference suppression by a link reduces the resources available to maximize the link's individual capacity. Thus, there is a fundamental trade-off between boosting individual link performance and reducing interference, which can increase spatial reuse and overall performance. In general, the diversity-multiplexing trade-off has been very well studied in the MIMO literature, but there is very little work on the diversity-multiplexing-suppression trade-off [8]. It is exactly this trade-off that we study in this paper.

To be specific, we consider the problem of maximizing throughput in a multihop MIMO network while accounting for variable-rate streams on individual MIMO links, and full interference suppression (both receiver-side and transmitterside suppression). Our method of handling link rates explicitly accounts for diversity and array gains on the links. We formulate the maximum-throughput problem as an integer linear program (ILP), which represents the first exact formulation of a MIMO network optimization problem with full interference suppression. We demonstrate the feasibility of computing exact optimal solutions to the maximum throughput problem by using CPLEX to solve the ILP problem on networks with up to 32 concurrently transmitting links and varying levels of interference. We also adapt a heuristic scheduling algorithm, which was developed for a related problem, to the one studied in this paper. The performance of the heuristic is shown to be in the range of $10 \%$ to $20 \%$ from optimal. We further show that the performance of the heuristic suffers due to its inability to fully capture the diversity-multiplexing-suppression tradeoff, which the ILP solution optimally handles. We also show that the use of interference suppression produces more than a multiplicative factor of 2 improvement in performance, as compared to optimizing individual MIMO links' performance without interference suppression.

\section{BACKGROUND}

\section{A. MIMO Networks}

We refer to a wireless network containing MIMO links as a MIMO network. The goal of this research is to use the MIMO resources available on the links to optimize overall network performance, rather than to separately optimize performance of individual links, which can lead to sub-optimal network performance. MIMO resources are antenna elements present at each end of a MIMO link. These antenna elements can be used for array gain, diversity gain, spatial multiplexing, and/or interference suppression [2]. The problem is to find the best operating point among these capabilities on each link so as to optimize some measure of network performance. We focus on maximizing aggregate network throughput, i.e. the aggregate network data rate across a set of simultaneous onehop communications. Thus, although the network is multihop, we do not consider aspects such as routing, bandwidth 
allocation, etc. Rather, we focus on the maximum throughput that can be achieved in the network in a single scheduling slot by optimizing the set of links that are scheduled in the slot and the use of resources on those links to maximize their aggregate data rate. Such a problem is typically referred to as a "one-shot scheduling" problem [9] and can form the basis for complete link scheduling solutions that schedule all links in the multi-hop network and account for factors such as routing and bandwidth allocation.

The use of MIMO antenna elements is typically modeled with degrees of freedom (DOFs). The DOFs available on a link characterize the number of independent streams that can be spatially multiplexed on the link, the amount of interference that can be suppressed by the link, and a trade-off between these two capabilities. A node with $k$ antenna elements has up to $k$ DOFs, which it can use for multiplexing and/or interference suppression. In the absence of interference, a link with $k_{t}$ DOFs at the transmitter and $k_{r}$ DOFs at the receiver can support up to $\min \left(k_{t}, k_{r}\right)$ spatially multiplexed streams. If DOFs are used for interference suppression, then the number of streams that can be supported on a link is reduced.

With a MIMO link, interference suppression can be done by the transmitter or by the receiver or both. To completely eliminate interference requires channel state information (CSI). Receivers can measure channels during transmission of probe sequences in order to collect CSI necessary both for interference suppression and for performance optimization of the channel. CSI can be fed back from receivers to transmitters or, if the channel is symmetric, transmitters can measure CSI by exchanging roles with receivers. We assume in this work that interference suppression can be done by both transmitters and receivers. However, the special cases of receiver-sidesuppression-only and transmitter-side-suppression-only can easily be modeled by simply assigning zero values to some variables in our linear program formulation.

The number of DOFs needed by a transmitter to suppress interference on a concurrent receiver is equal to the number of streams that are spatially multiplexed on the receiver's link. Similarly, the number of DOFs needed by a receiver to suppress interference from a concurrent transmitter is equal to the number of streams that are spatially multiplexed on the transmitter's link. Assume that a node $i$ has $k$ DOFs, spatially multiplexes $s_{i}$ streams on its link, and suppresses interference with other nodes $j_{1}, \ldots, j_{n}$, carrying $s_{j_{l}}$ streams respectively. Then, the following inequality must be satisfied, whether $i$ is a transmitter or a receiver:

$$
s_{i}+\sum_{l=1}^{n} s_{j_{l}} \leq k
$$

\section{B. Network and Interference Models}

We consider multi-hop networks consisting of a set of links $L=\left\{l_{1}, l_{2}, \ldots, l_{m}\right\}$, where each $l_{i}$ is a transmitter-receiver pair $\left(t_{i}, r_{i}\right)$. Uni-directional links are allowed. Any wireless propagation model can be used to compute the set of links, given a set of node positions, or the set of links can simply be given as input. Communication rates achievable on the links are discussed in the next subsection. We assume that all links use the same wireless channel. Joint optimization across multiple channels is not considered. However, if assignments of links to (non-overlapping) channels is done separately, then our optimization procedure can be performed independently within each channel.

The primary limitation on concurrent wireless transmissions in the same channel is interference. Here, we consider both primary interference, wherein a single wireless node is restricted from participating in multiple simultaneous communications ${ }^{1}$, and secondary interference, which is interference between two wireless links that do not have any node in common. A set of links is primary-interference-free if and only if every node in the network is part of at most one link in the set.

For secondary interference, we adopt a simple binary interference model wherein two links either interfere completely or not at all. Thus, we define a directed conflict graph $C G=$ $(L, E)$, where the vertex set $L$ is the set of links to be scheduled, and directed edge $\left(l_{i}, l_{j}\right) \in E$ if transmitter $t_{i}$ causes interference at receiver $r_{j}{ }^{2}$ Again, we do not assume any specific underlying interference model, e.g. interference is not necessarily specified by a simple interference range nor do the conflicts have to be symmetric. We simply assume that the conflicts between links are known. The conflict graph can be computed from the set of links and the node positions by specifying an underlying (binary) interference model or the conflict graph can simply be given as input.

Admittedly, modeling interference as a binary phenomenon is a simplification of reality, and using more complex SINRbased interference models as is done in some non-MIMO wireless scheduling (e.g., [4]) would be preferable. However, including SINR considerations into network MIMO models is an open problem, which requires developing novel PHY layer MIMO models, and is beyond the scope of this paper. For this reason, modeling interference as a binary phenomenon is a common assumption in MIMO network literature [3], [10].

\section{Rates and Streams}

The data rate on an individual MIMO link is determined by the characteristics of the channel in between the transmitter and receiver, the numbers of DOFs used at the transmitter and the receiver for multiplexing, and the number of multiplexed streams. We model this with a rate function $d r(i, t, r, s)$, which gives the rate on the link $i=\left(t_{i}, r_{i}\right)$ when $t_{i}$ uses $t$ DOFs for multiplexing, $r_{i}$ uses $r$ DOFs multiplexing, and $s$ independent streams are spatially multiplexed on the link. ${ }^{3}$ Note that the above rate is computed in absence of interference, since in our approach interference is always completely removed

\footnotetext{
${ }^{1}$ Here, we assume each node is equipped with only a single radio.

${ }^{2}$ Note that the conflict graph could easily be defined to account for both primary interference and secondary interference. However, since the conflict graph is primarily used to make decisions about how to suppress secondary interference, we restrict it to that type herein.

${ }^{3}$ Although the data rate is also dependent on transmission power, we assume all nodes transmit at a fixed power and hence we do not consider the added dimension of power control in the optimization problem formulation.
} 
by allocating MIMO resources and performing interference suppression. We do not make any assumption on the rate function, i.e. it can be an arbitrary function. Note that, if the channel between $t_{i}$ and $r_{i}$ is random (as is the case with Rayleigh fading channels, for example), the rate on the link is also a random variable. In this case, we interpret $d r$ as the expected data rate, which can also be thought of as the long-term rate on the link if its channel characteristics change dynamically and at random.

Note that the rate functions as specified above are approximations to the actual rates, which depend on the instantaneous channel characteristics and the MIMO weights that are chosen by the transmitter and receiver. In Section V, we discuss how we approximated the rate functions in order to carry out the simulation experiments reported in that section. While we believe those rates to be reasonable approximations, which capture the essence of the diversity-multiplexing-suppression trade-off, if more accurate techniques for approximating data rates are developed, they could easily be plugged into our problem formulation.

Table II shows representative rates for one link used in our later evaluations. This table illustrates the complex nature of the optimizations involved in our problem setting. The amount of interference generated by a link $l$ is dependent on the number of streams that are spatially multiplexed on $l$ (every other link that wishes to suppress interference on or from $l$ must allocate one DOF for every stream on $l$ ). However, while interference increases linearly with the number of streams, there is a law-of-diminishing-returns effect on data rate as the number of streams increases. Note in the table that going from 1 to 4 streams increases the link data rate by at most a factor of $\frac{119.25}{56.31} \approx 2.12$. Thus, even if a link has extra DOFs available, the overall network performance might benefit if the link uses those DOFs for array and diversity gains, rather than for increasing the number of streams it transmits. For example, a link with the characteristics in Table II that has 3 DOFs available at both transmitter and receiver could use those DOFs to transmit 2 streams, thereby achieving almost as high a rate as with 3 streams but generating less interference in the network. Clearly, the shape of the rate function has a strong influence on the nature of the optimal solution and it is, therefore, critical to know the rate function values when trying to optimize network performance.

\section{RELATED WORK}

The vast amount of work on integrating MIMO with the networking stack has focused on the MAC layer. Here, we are interested in network-wide optimization. We also do not consider cooperative MIMO using antenna arrays distributed across multiple nodes. Cooperative MIMO requires tight synchronization between transmissions on different nodes, which provides extreme challenges for high data rate communications. We consider the use of MIMO techniques on nodes of an individual link, where tight synchronization is possible and MIMO techniques are already widely deployed [12]. We also consider the use of interference suppression through
MIMO, which involves multiple links but does not require synchronization of communications [1].

It has only been fairly recently that MIMO networking research has considered network throughput optimization [3], [5], [10], [11], [13], [14], [16], [17], [18], [19]. A number of these papers present formal network optimization problem formulations [3], [5], [10], [13], [19]. Sundaresan, et al., give a probabilistic throughput formulation but do not explicitly consider interference constraints [19]. Bhatia and Li [3] and Hamdaoui and Shin [10] include constraints that account for both transmitter-side and receiver-side suppression but they are based on necessary but not sufficient feasibility conditions. Hamdaoui, Shin, and Maiya extend the problem formulation to include multiple frequency bands [11]. Chu and Wang [5] include constraints that are sufficient but not necessary for feasibility and they assume receiver-side suppression only. Liu, Hou, and Sherali jointly consider the problems of power control and routing with a maximum throughput objective through a network flow formulation [13]. Interference constraints are not considered. Mumey, Tang, and Hahn give an approximation algorithm for joint stream control and scheduling, where only receiver-side interference suppression is considered. In [17], we solved the problem of maximizing throughput within a single time slot exactly, for the special case where all links reside within a single collision domain, all nodes have the same antenna array size, and all streams have the same rate. In [14], Liu, Shi, and Hou presented an interference cancellation approach for multi-hop MIMO networks, which is based on a sufficient (but not necessary) condition for feasibility. This work also assumes that data rate increases linearly with the number of streams on a link and, therefore, does not account for diversity and array gains.

The most significant difference between our work and prior work is that we use rate functions on a link, which specifically consider the dependence of channel capacity on the numbers of DOFs used for transmitting and receiving. This allows array and diversity gains to be factored into MIMO resource allocation decisions, which is necessary to fully exploit the diversity-multiplexing-suppression trade-off.

\section{LiNEAR PROGRAM Formulation}

We consider a set $L=\left\{l_{1}, \ldots, l_{l}\right\}$ of MIMO links to schedule. For each link $l_{i}=\left(t_{i}, r_{i}\right), t_{i}$ and $r_{i}$ denote the transmitter and receiver node of the link, respectively. The number of antenna elements at the $i$-th transmitter (respectively, receiver) is denoted $k_{i}^{t}$ (respectively, $k_{i}^{r}$ ). Note that it is possible that different links are incident in the same wireless node, i.e., we might have $t_{i}=t_{j}$, or $r_{i}=r_{j}$, or $t_{i}=r_{j}$, for some $i \neq j$. In the following, we denote by $V=\left\{v_{1}, \ldots, v_{n}\right\}$ the set of wireless nodes that make up the transmitters and receivers of link set $L$ (note that $n \leq 2 l$ ). For a given wireless node $v \in V$, we denote by $L(v)$ the set of indices of links for which $v$ is either transmitter or receiver. Formally,

$$
L(v)=\left\{i \in\{1, \ldots, l\} \mid\left(t_{i}=v\right) \vee\left(r_{i}=v\right)\right\} .
$$


For a conflict graph $C G=(L, E)$, we define interference sets as follows. The set of links interfered by a link $l_{i}$, i.e., the set of links corresponding to the outgoing neighbors of $l_{i}$ in $C G$, is denoted by $I^{t}(i)$. The set of links whose transmitters cause interference at $r_{i}$, i.e., the set of links corresponding to the incoming neighbors of $l_{i}$ in $C G$, is denoted by $I^{r}(i)$.

Antenna elements at nodes $t_{i}, r_{i}$ provide degrees of freedom (DOFs) that can be used to transmit data on link $l_{i}$ and/or to suppress interference to or from links in $I^{t}(i)$ or $I^{r}(i)$. In particular, $t_{i}$ can use DOFs to suppress interference at a receiver $r_{j}$ with $j \in I^{t}(i)$, or $r_{i}$ can use DOFs to suppress interference generated by transmitter $t_{j}$ with $j \in I^{r}(i)$. The total DOFs available at $t_{i}$ (respectively, $r_{i}$ ) for multiplexing and interference suppression is given by $k_{i}^{t}$ (respectively, $k_{i}^{r}$ ).

The data rate function for $l_{i}$ is denoted by $d r_{i t r s}$, with $t=1, \ldots, k_{i}^{t}, r=1, \ldots, k_{i}^{r}$, and $s=1, \ldots, \min (t, r)$, which gives the data rate on link $l_{i}$ when $t$ DOFs are used for transmission at $t_{i}, r$ DOFs are used for reception at $r_{i}$, and $s$ streams are spatially multiplexed on $l_{i}$. The presented results are valid for arbitrary values of the $d r_{i t r s}$ 's, which are considered as input values to the problem.

In the following, we use indicator variables to represent usage of DOFs at the transmitters and receivers of the $l$ links. For each pair $i, j$ of links in $L$, we have two sets of variables $\gamma_{i j m}$ and $\delta_{i j n}$, with $m=1, \ldots, k_{i}^{t}$ and $n=1, \ldots, k_{i}^{r}$. $\gamma_{i j m}$ is 1 if transmitter $t_{i}$ is using $m$ DOFs to suppress interference at $r_{j}$, and 0 otherwise. Similarly, $\delta_{i j n}$ is 1 if receiver $r_{i}$ is using $n$ DOFs to suppress interference from $t_{j}$. Furthermore, we use variables $x_{\text {itrs }}$ (called resource allocation variables), for $i=1, \ldots, l, t=1, \ldots, k_{i}^{t}, r=1, \ldots, k_{i}^{r}$, and $s=1, \ldots, \min (t, r)$, where $x_{i t r s}=1$ if $t$ DOFs are used for transmission along $l_{i}$ at $t_{i}, r$ DOFs are used for reception along $l_{i}$ at $r_{i}$, and $s$ streams are spatially multiplexed on $l_{i}$, and $x_{i t r s}=0$, otherwise. Finally, we use variables $z_{i}$, for $i=1, \ldots, l$, where $z_{i}=1$ if link $i$ is inactive, and 0 otherwise. The notation used is summarized in Table I.

\begin{tabular}{|c|l|}
\hline$L=\left\{l_{1}, \ldots, l_{l}\right\}$ & set of $l$ links to be scheduled \\
$V=\left\{v_{1}, \ldots, v_{n}\right\}$ & set of wireless nodes \\
$L(v)$ & set of links incident into node $v$ \\
$t_{i}, r_{i}$ & transmitter and receiver of link $l_{i}$ \\
$k_{i}^{t}, k_{i}^{r}$ & number of antenna elements (also DOFs) at $t_{i}, r_{i}$ \\
$I^{t}(i)$ & set of links interfered by $t_{i}$ \\
$I^{r}(i)$ & set of links whose transmitters interfere with $r_{i}$ \\
$d r_{i t r s}$ & link data rate when $t_{i}$ uses $t$ DOFs to transmit \\
& along $l_{i}, r_{i}$ uses $r$ DOFs to receive, \\
& and $s$ streams are sent on $l_{i}$ \\
$\gamma_{i j m}$ & $=1$ iff $m$ DOFs are used by $t_{i}$ to suppress \\
& interference at $r_{j}$ \\
$\delta_{i j n}$ & $=1$ iff $n$ DOFs are used by $r_{i}$ to suppress \\
& interference from $t_{j}$ \\
$x_{i t r s}$ & $=1$ iff $t_{i}$ uses $t$ DOFs to transmit along $l_{i}$, \\
& $r_{i}$ uses $r$ DOFs to receive, and $s$ streams \\
& are sent on $l_{i}$ \\
& $=1$ iff link $l_{i}$ is inactive \\
\hline
\end{tabular}

TABLE I

NOTATION USED IN THE ILP PROBLEM FORMULATION.

On the next page is the formal specification of our throughput maximization problem, called OSTM (OneShotThroughputMaximization), as an integer linear program. Note that the ILP formulation of OSTM is not trivial, as the direct formulation of several constraints, e.g., those related to interference suppression, gives rise to non-linear inequalities. To get around this problem, we had to introduce indicator variables in several places, e.g., the $x_{i t r s}$ resource allocation variables, which allowed us to remove the non-linearities at the expense of increasing the overall number of variables in the ILP formulation.

The inputs to the linear program are the link set $L$, the wireless node set $V$, the data rate values $d r_{i t r s}$, the numbers of available antenna elements ( $k_{i}^{t}$ and $k_{i}^{r}$ values) at each node, and the interference sets $I^{t}(i)$ and $I^{r}(i), \forall i$. The outputs of the linear program are a set of resource allocation variables $\left(x_{i t r s}\right.$ 's), and a set of DOF assignments ( $\gamma_{i j m}$ 's and $\delta_{i j n}$ 's) that completely suppress interference for the resource allocation.

The objective function states that we want to maximize the sum of link data rates. Constraint (5) states that the sum of the resource allocation variables and the link activation variable relative to link $l_{i}$ must be equal to 1 which, coupled with Constraints (1) and (2), ensures that exactly one amongst the $x_{i t r s}$ variables and $z_{i}$ must be 1 . Constraints (3) and (4) state that the values of the $\gamma$ and $\delta$ variables must be $0 / 1$.

Constraint (6), coupled with Constraint (2), accounts for primary interference, dictating that at most one of the $x_{i t r s}$ variables incident into a wireless node $v$ must be 1 .

Constraints (7) through (11) are notational definitions. Constraint (7) defines $u_{i}^{t}$ to be equal to the number of DOFs used at the transmitter side of link $l_{i}$ (note that $u_{i}^{t}=0$ when all $x_{i t r s}$ 's are 0). Similarly, Constraint (8) defines $u_{i}^{r}$ to be equal to the number of DOFs used at the receiver side of link $l_{i}$. Constraint (9) defines $s_{i}$ to be equal to the number of streams being transmitted along link $l_{i}$ (note that $s_{i}=0$ when all $x_{i t r s}$ 's are 0). Constraints (10) and (11) define $a_{i j}^{t}$ and $a_{i j}^{r}$ to be equal to the number of DOFs used by $t_{i}$ to suppress interference at $r_{j}$, and by $r_{i}$ to suppress interference from $t_{j}$, respectively (note that $a_{i j}^{t}=0$ when all the $\gamma_{i j m}$ 's are 0 , and similarly for $a_{i j}^{r}$ with the $\delta_{i j n}$ 's).

Constraints (12) and (13) are called the DOF constraints, and state that the sum of the number of DOFs used for transmission/reception along $l_{i}$ and the number of DOFs used at the transmitter/receiver for suppressing interference must not exceed the number of available antenna elements.

Finally, Constraints (14), (15), and (16) are the interference constraints. The first two interference constraints state that, for each link $l_{j}$ interfered by link $l_{i}$, either $s_{j}$ DOFs must be used by $t_{i}$ or $s_{i}$ DOFs must be used by $r_{j}$ to suppress the interference. However, these constraints only apply if both $l_{i}$ and $l_{j}$ are active. By choosing $M$ sufficiently large (it is enough to set $M$ larger than the maximum number of antenna elements available at a wireless node), the constraints are automatically satisfied whenever either link is inactive. The third interference constraint imposes that, when two links $l_{i}$ and $l_{j}$ interfere, the summation of the $\gamma$ variables at $t_{i}$ and of the $\delta$ variables at $r_{j}$ does not exceed 1 , thus ensuring that interference suppression is entirely done at either $t_{i}$ or $r_{j}$. 


\section{Numerical Evaluations}

$\begin{array}{rc}\max & \sum_{i=1}^{l} \sum_{t=1}^{k_{i}^{t}} \sum_{r=1}^{k_{i}^{r}} \sum_{s=1}^{\min \{t, r\}} x_{i t r s} \cdot d r_{i t r s} \\ \forall i & z_{i} \in\{0,1\} \\ \forall i, t, r, s & x_{i t r s} \in\{0,1\} \\ \forall i, j, m & \gamma_{i j m} \in\{0,1\} \\ \forall i, j, n & \delta_{i j n} \in\{0,1\}\end{array}$

$\forall i \quad z_{i}+\sum_{t=1}^{k_{i}^{t}} \sum_{r=1}^{k_{i}^{r}} \sum_{s=1}^{\min \{t, r\}} x_{i t r s}=1$

$\forall v \in V \quad \sum_{i \in L(v)} \sum_{t=1}^{k_{i}^{t}} \sum_{r=1}^{k_{i}^{r}} \sum_{s=1}^{\min \{t, r\}} x_{i t r s} \leq 1(6)$

$\forall i \quad u_{i}^{t}=\sum_{t=1}^{k_{i}^{t}} \sum_{r=1}^{k_{i}^{r}} \sum_{s=1}^{\min \{t, r\}} t \cdot x_{i t r s}$

$\forall i \quad u_{i}^{r}=\sum_{r=1}^{k_{i}^{r}} \sum_{t=1}^{k_{i}^{t}} \sum_{s=1}^{\min \{t, r\}} r \cdot x_{i t r s}$

$\forall i$

$$
s_{i}=\sum_{t=1}^{k_{i}^{t}} \sum_{r=1}^{k_{i}^{r}} \sum_{s=1}^{\min \{t, r\}} s \cdot x_{i t r s}
$$$$
a_{i j}^{t}=\sum_{m=1}^{k_{i}^{t}} m \cdot \gamma_{i j m}
$$

$\forall i, j$

$a_{i j}^{r}=\sum_{n=1}^{k_{i}^{r}} n \cdot \delta_{i j n}$

$u_{i}^{t}+\sum_{j \in I^{t}(i)} a_{i j}^{t} \leq k_{i}^{t}$

$u_{i}^{r}+\sum_{j \in I^{r}(i)} a_{i j}^{r} \leq k_{i}^{r}$

$\forall i, \forall j \in I^{t}(i)$

$M \cdot\left(z_{i}+z_{j}\right)+a_{i j}^{t}+M \cdot a_{j i}^{r} \geq s_{j}$

$\forall i, \forall j \in I^{t}(i)$

$M \cdot\left(z_{i}+z_{j}\right)+a_{j i}^{r}+M \cdot a_{i j}^{t} \geq s_{i}$

$\sum_{m=1}^{k_{i}^{t}} \gamma_{i j m}+\sum_{n=1}^{k_{j}^{r}} \delta_{j i n} \leq 1$

To the best of our knowledge, this is the first exact formulation of a network-level MIMO scheduling problem with interference suppression. In fact, similar formulations presented in [3], [5], [10], [14], although considering more general problems in which multi-hop flows and routing are considered, express only necessary conditions or only sufficient conditions for interference suppression, but not both. Thus, the presented results must be considered as upper bounds or lower bounds to the actual achievable throughput. On the contrary, our ILP formulation for OSTM is exact, i.e., it is guaranteed to return an feasible solution, which is optimal under the stated assumptions. Furthermore, differently from [3], [5], [10], [14], our formulation is general enough to encompass an additional reality of MIMO systems, namely link rates that depend on DOF allocations of the transmitter and receiver, rather than simply on the number of streams transmitted.

It should be noted that the complete linear program formulation presented herein has several simpler MIMO subproblems embedded within it. For example, in the stream allocation problem defined in [18], a primary-interference-free link set is given. This version of the problem is arrived at by removing Constraint (6), which enforces primary interference restrictions. In [18] this problem was defined (informally) and heuristic algorithms for single-collision-domain networks were studied. Here, we study the exact optimal solution for multihop networks. Also, removing the $\gamma_{i j m}$ variables and modifying or removing the associated constraints yields a version of the problem for the case where interference suppression is done only at the receiver side. In a similar way, removing the $\delta_{i j n}$ variables yields the transmitter-side-suppression-only case.
The ILP defined in the previous section has been used to numerically compute the optimal solution to the OSTM problem. Numerical evaluations have been done running CPLEX on an Intel i7 920 (Quad core) machine with 6 GB of RAM.

\section{A. Evaluation Set-up}

One of the ILP inputs is the rate function $d_{i t r s}$, representing, for each possible link $i$, the aggregate rate on the link when $t$ DOFs are used for transmission by $t_{i}, r$ DOFs are used for reception at $r_{i}$, and $s \leq \min \{t, r\}$ streams are transmitted on the link. Input rates have been numerically calculated by modeling the radio channel as an idealized rich scattering static environment. The resulting channel model corresponds to a quasi-static Rayleigh fading channel model, according to which the channel has i.i.d., complex, zero mean, unit variance elements as described in [6]. The gain of each channel matrix was calculated using Friis transmission equation and the log-distance path loss model with exponent of 3 [15]. Since in our optimization framework, we assume perfect channel state information is available at both receivers and transmitters, optimal power allocation through water filling at transmitters has been considered when computing rates, which are computed using Shannon's capacity formula with the optimal power values [7].

In order to approximate the data rate due to the use of some DOFs for interference suppression, we first perform antenna selection and then find the optimal data rate as described above. We perform antenna selection by picking the $t$ transmit elements and the $r$ receive elements that maximize the data rate of the link. We then calculate the rates for the case of $1 \leq s \leq \min (t, r)$ streams by allocating power through the best $s$ eigenmodes of the $t \times r$ channel.

An example of input rates obtained for a certain transmitterreceiver pair for different values of $t, r$, and $s$, and for $k_{i}^{t}=k_{i}^{r}=4$ are reported in Table II. The benefits of array and diversity gains are apparent. When a single stream is sent using $1 \mathrm{DOF}$ at both transmitter and receiver, a rate of $56.31 \mathrm{Mb} / \mathrm{sec}$ is obtained. The rate with a single stream increases to $76.68 \mathrm{Mb} / \mathrm{sec}$ with $2 \mathrm{DOFs}$ at both ends and to $87.71 \mathrm{Mb} / \mathrm{sec}$ with 4 DOFs. Spatial multiplexing is also effective in increasing aggregate rate on the link: the aggregate rate on the link increases from $56.31 \mathrm{Mb} / \mathrm{sec}$ (1 stream sent with $1 \mathrm{DOF}$ at both sides of the link) to $119.25 \mathrm{Mb} / \mathrm{sec}$ (4 streams sent with 4 DOFs at both sides of the link). As noted earlier, the rate increase is sub-linear with respect to the number of streams: a factor of 2.12 increase in rate is achieved with a factor of 4 increase in the number of streams.

In all evaluations, wireless nodes (the link endpoints) are arranged in a regular grid, with inter-node distance normalized to 1. Regular deployment of wireless nodes, coupled with a notion of interference range (see below), allows precise control of the amount of interference generated in the wireless medium, which is a critical factor in the resulting throughput. In one group of simulations, we predetermine sets of primaryinterference-free links that form maximum matchings from all 


\begin{tabular}{|c|cccc|}
\cline { 2 - 5 } \multicolumn{1}{c|}{} & \multicolumn{4}{|c|}{$(t, r)$} \\
\hline$s$ & $(1,1)$ & $(2,2)$ & $(3,3)$ & $(4,4)$ \\
\hline 1 & 56.31 & 76.64 & 85.01 & 87.71 \\
\hline 2 & - & 82.25 & 104.96 & 116.26 \\
\hline 3 & - & - & 105.06 & 119.25 \\
\hline 4 & - & - & - & 119.25 \\
\hline
\end{tabular}

TABLE II

LINK RATES OF A SAMPLE LINK (IN $\mathrm{Mb} / \mathrm{sec}$ ) FOR DIFFERENT NUMBERS OF DOFS AND STREAMS $\left(k_{i}^{t}=k_{i}^{r}=4\right)$

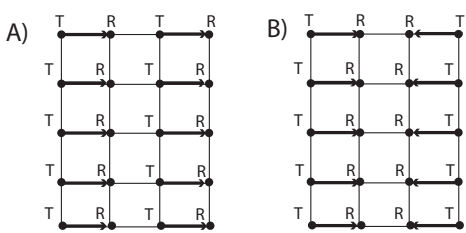

Fig. 1. Example of type 1 (left) and type 2 (right) link sets with 10 links of the grid links and determine the optimal solution for those sets. In a second group of simulations, we choose random link sets from all of the grid links and evaluate the optimal solution for each random set. In one sub-case, we choose a specified number of arbitrary grid links at random, and the chosen link set is not necessarily primary-interference-free. In a second sub-case, we choose a specified number of grid links at random while maintaining the primary-interference-free requirement.

The conflict graph is based on an interference range, denoted by range $_{I}$. An edge between the vertex corresponding to link $l_{i}$ and the vertex corresponding to $l_{j}$ in the conflict graph is inserted if and only if wireless node $r_{j}$ is within

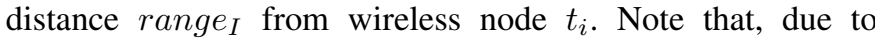
different distances between $t_{i}, r_{j}$ and $t_{j}, r_{i}$, the fact that edge $\left(l_{i}, l_{j}\right)$ is in the conflict graph does not necessarily imply that the reverse edge is also in the conflict graph. In the following, in order to clarify presentation, we consider only discrete interference levels from 1 to 9 , corresponding to different interference ranges as detailed in Table III. The same levels are used also for communication range. Unless otherwise specified, the communication level is set to 1 in the following.

\begin{tabular}{|c|c|c|c|}
\hline level & distance & level & distance \\
\hline \hline 1 & 1.0 & 6 & 3.0 \\
2 & 1.414 & 7 & 3.162 \\
3 & 2.0 & 8 & 3.605 \\
4 & 2.236 & 9 & 4.242 \\
5 & 2.828 & & \\
\hline
\end{tabular}

TABLE III

INTERFERENCE RANGES FOR DIFFERENT INTERFERENCE LEVELS

\section{B. Optimal Solution with Predetermined Link Sets}

In the first set of evaluations, we considered primary interference free link sets that are pre-selected from among the grid links. In particular, two types of link sets were considered, called type 1 and type 2 link sets (see Figure 1). Both of these link sets correspond to maximum matchings of the communication graph of the grid and they are, therefore, maximum-sized primary-interference-free link sets. The only difference between the two link sets is in the link orientation: links in the same row have the same orientation in type 1 link sets, while the orientations alternate in type 2 link sets.

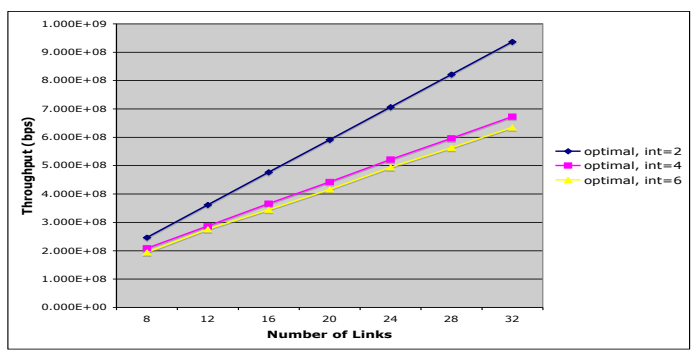

Fig. 2. Optimal aggregate throughput for type 1 link sets and different interference levels $\left(k_{i}^{t}=k_{i}^{r}=4, \forall i\right)$

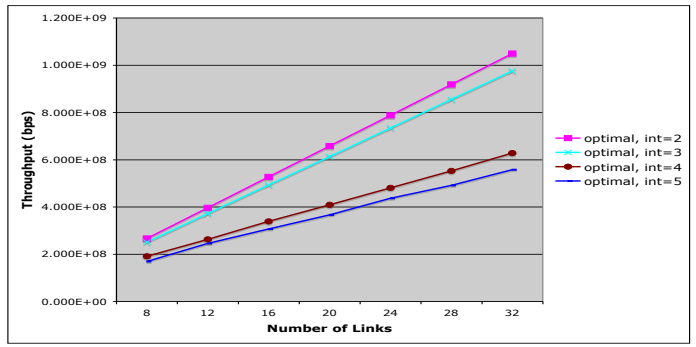

Fig. 3. Optimal aggregate throughput for type 2 link sets and different interference levels $\left(k_{i}^{t}=k_{i}^{r}=4, \forall i\right)$

For each link set type, we used CPLEX to compute the optimal solution of OSTM using the ILP formulation presented earlier. We calculated the optimal value for increasing grid sizes and for different interference levels. The results are shown in Figure 2 for type 1 link sets and in Figure 3 for type 2 link sets. For type 1 link sets, the conflict graph does not change when going from interference level 2 to level 3 and from interference level 4 to level 5. Thus, we have shown only interference levels 2, 4, and 6 for this case.

In terms of the behavior of the optimal solutions, we see that for both types of link sets, the throughput increases linearly with the number of links for a fixed interference level. This is as expected, because for a fixed interference level, a constant number of links fall within a single interference range with the grid topology. As the interference level increases, the magnitude of the aggregate throughput decreases as does the slope of the line representing the throughput. The decrease in throughput that occurs as the interference level increases is not uniform. This is because the number of new receivers that are added to a single interference range around a given transmitter does not increase uniformly as the interference level is increased. This causes the same non-uniform increase in the degree of the conflict graph, which is the primary determining factor for the throughput in this situation. There is no clear advantage to type 1 or type 2 link sets: for some interference levels, type 1 link sets are slightly better and for other levels, type 2 sets are slightly better.

\section{Optimal Solution with Random Link Sets}

In this set of evaluations, we computed the optimal throughput for randomly selected link sets. In the first evaluations, 32 links were randomly selected out of the 224 possible links in an $8 \times 8$ grid. These links are not necessarily primaryinterference-free and so this corresponds to the full version of the MIMO link scheduling problem. The average optimal throughput over ten random link sets was evaluated for each 


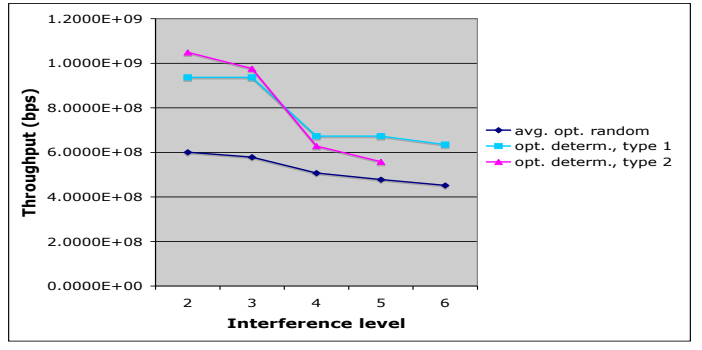

Fig. 4. Optimal aggregate throughput for random link sets (with primary interference) and type 1 and 2 link sets $\left(k_{i}^{t}=k_{i}^{r}=4, \forall i\right)$

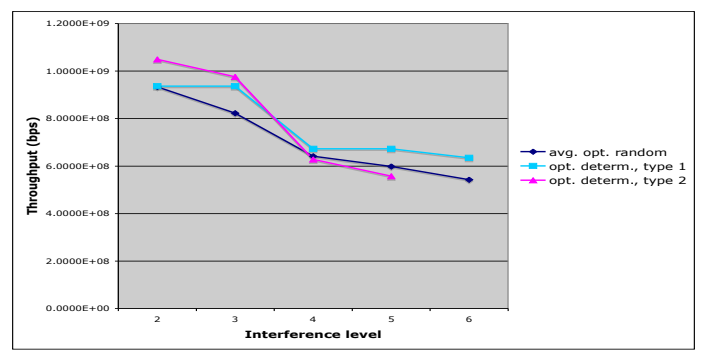

Fig. 5. Optimal aggregate throughput for random link sets (without primary interference) and type 1 and 2 link sets $\left(k_{i}^{t}=k_{i}^{r}=4, \forall i\right)$

interference level. For comparison, the corresponding optimal values for the pre-selected type 1 and type 2 link sets are also shown. The results are shown in Figure 4.

In terms of the feasibility of calculating the optimal solution, these results demonstrate that the ILP solution complexity is primarily dependent on the number of links that are input to CPLEX and is not significantly impacted by whether the link sets are primary interference free or not. When the link set has primary interference, Constraint (6) must be evaluated, while for primary-interference-free link sets, it is automatically satisfied. Addition of this constraint did not significantly increase convergence time for CPLEX.

The results show that including links with primary interference degrades the quality of the optimal solution, particularly for small interference levels. In these cases, primary interference is dominant and the throughput of primary-interferencefree link sets is more than $60 \%$ higher than ones with primary interference. As interference level increases, secondary interference increases and the difference between the optimal solutions for the two cases is reduced.

In a second set of experiments, we randomly selected links in sequence considering only those links that had no primary interference with already chosen links. In this manner, we were able to select random primary-interference-free link sets with 30 links in them. Again, 10 different random link sets were generated and the results averaged. The results of these computations are shown in Figure 5. For comparison, the optimal results from type 1 and type 2 link sets (with 32 links each) are shown also. The results show that the random primary-interference-free link sets perform almost as well as the pre-selected type 1 and type 2 sets. Performance seems to be determined primarily by the number of primaryinterference-free links, rather than the specific structure of the corresponding matching. By pre-selecting the links, we were able to generate maximum matchings of 32 links for these regular networks, whereas when randomly generating matchings, 30 links was the maximum number that could be consistently generated.

\section{Computational Time}

Clearly, since the worst-case complexity of general ILP problems is exponential (unless $\mathbf{P}=\mathbf{N P}$ ), the time needed to compute the optimal solution is an issue. In particular, what is interesting to evaluate is the average time needed to compute a solution of the various problem instances considered.

We first observe that, in case of grid networks of either type 1 or 2, optimal solutions can be computed quite efficiently for relatively low values of the interference range and 32 or fewer active links. As the interference range increases, the number of interference constraints increases as well, with a negative impact on computational time - see Table IV referring to the case of type 1 networks. Recall that with an interference level of 3 , the interference range is twice the communication range, which is the most common assumption in network evaluations. For this case, less than two seconds of computation time are required, even with 32 active links.

\begin{tabular}{|c|c|c|c|}
\hline Active Links & int $=2$ & int $=3$ & int $=4$ \\
\hline \hline 8 & 0.12 & 0.12 & 1.13 \\
12 & 0.12 & 0.13 & 1.97 \\
16 & 0.22 & 0.2 & 5.12 \\
20 & 0.59 & 0.62 & 14.56 \\
24 & 0.91 & 0.95 & 49.18 \\
28 & 1.21 & 1.21 & 883.02 \\
32 & 1.64 & 1.72 & 3812.43 \\
\hline
\end{tabular}

TABLE IV

TIME NEEDED TO COMPUTE THE OPTIMAL SOLUTION (IN $s e c$ ) FOR TYPE 1 GRID NETWORKS WITH DIFFERENT INTERFERENCE LEVELS

With random networks, the time needed to compute the optimal solution increases with respect to the case of grid networks with comparable number of links and interference range - see Table V. This is likely due to the less structured shape of the optimal solution in case of random networks, as opposed to very regular optimal solutions obtained with grid networks (see Figure 8 later in this section). However, we emphasize that computing the optimal solution for networks of size as large as 30-32 links is feasible even for random networks. Referring to the computational times reported in Table V, we observe that instances with 32 links can be solved faster than those with 30 links. As described earlier, in the 30 link instances, all links were pre-selected to be free of primary interference. Hence, each of them can be considered as a good candidate link in the optimal solution. On the other hand, the 32 link instances included links possibly subject to primary interference. Since links subject to primary interference exclude each other from the optimal solution, the number of good candidate links for the optimal solution are indeed less (on average) than in the 30 link instances. This observation highlights that what influences computational time is not merely the number of links to be considered, but also their mutual primary and secondary interference relationships. 


\begin{tabular}{|c|c|c|c|c|}
\hline Links & int $=2$ & int $=3$ & int $=4$ & int $=5$ \\
\hline \hline 30 & 8 & 836 & 1233 & 5070 \\
32 & 12 & 173 & 322 & 955 \\
\hline
\end{tabular}

TABLE V

TIME NEEDED TO COMPUTE THE OPTIMAL SOLUTION (IN $s e c$ ) FOR RANDOM NETWORKS WITH DIFFERENT INTERFERENCE LEVELS

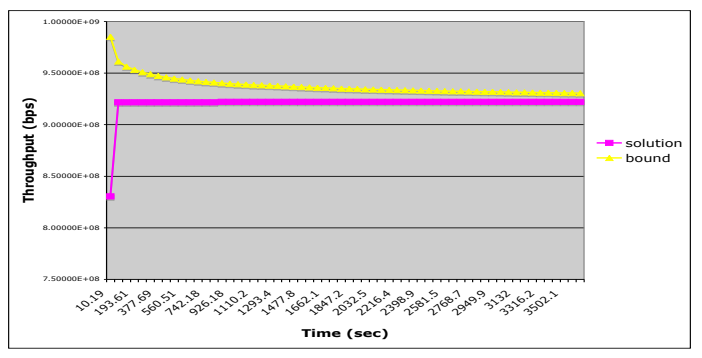

Fig. 6. CPLEX lower and upper bounds vs. time: slow-converging case $\left(k_{i}^{t}=\right.$ $\left.k_{i}^{r}=8, \forall i\right)$

Finally, we attempted to solve the ILP formulation when all nodes have 8 antenna elements instead of 4 . These experiments were only partially successful. In many cases, CPLEX converged fairly quickly (within a few hundred seconds) to the optimal solution. In some cases, however, convergence was much slower and in other cases memory usage rose dramatically preventing an optimal solution from being found. Figure 6 shows the lower and upper bounds calculated by CPLEX over time for a slow-converging DOF=8 case for 24 random links chosen from an $8 \times 8$ grid with an interference level of 2. In this case, CPLEX found a solution guaranteed to be slightly more than $1 \%$ away from optimal within 10 minutes but took almost one hour to complete the search.

\section{E. Comparison of Optimal and Sub-Optimal Solutions}

In this section, motivated by the fact that computing the optimal OSTM solution is feasible only for networks of moderate size, we compute several sub-optimal solutions and compare them to optimal. The first comparison focuses on a heuristic scheduling algorithm for multihop MIMO networks, which we refer to as Algorithm StreamMaxRate-Multihop (SMR-M). Algorithm SMR-M is based on Algorithm StreamMaxRate from [18], which is a type of gradient-descent heuristic scheduling algorithm for single-collision-domain MIMO networks, which accounts for arbitrary rate functions on links. Algorithm SMR-M extends Algorithm SMR to work in multihop networks and also executes the algorithm on 10 random link orderings and selects the best solution of the 10. The second comparison focuses on a situation where MIMO resources are used for spatial multiplexing, array gain, and diversity gain, but not for interference suppression. This corresponds to a situation where links are optimized individually, i.e. no multilink optimization of resource usage is performed.

Comparison with Algorithm SMR-M:

Figure 7 compares the optimal solution against the solution found by Algorithm SMR-M for type 1 link sets. The results for type 2 link sets were similar and are omitted due to

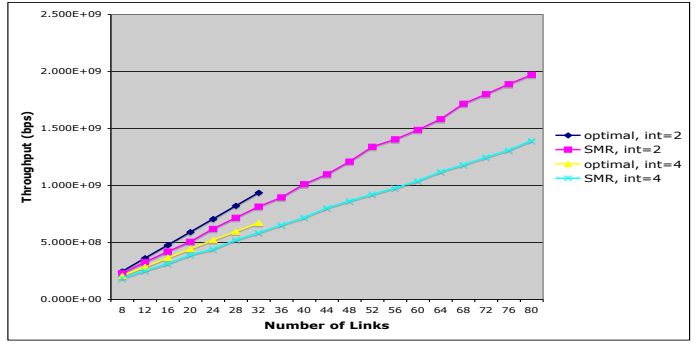

Fig. 7. Aggregate throughput for Algorithm StreamMaxRate-Multihop compared to optimal for type 1 link sets $\left(k_{i}^{t}=k_{i}^{r}=4, \forall i\right)$

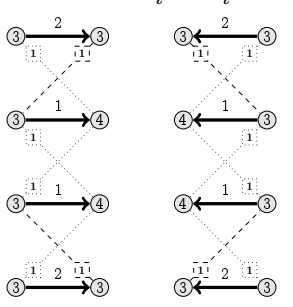

Fig. 8. Example of optimal solution ( $4 \times 4$ grid, int. level $2, k_{i}^{t}=k_{i}^{r}=4, \forall i$ ) space limitations. The results show that Algorithm SMR$\mathrm{M}$ can produce feasible but non-optimal solutions for large numbers of links. We computed the solutions for up to 80 links with SMR-M, but could easily have gone higher. One execution of SMR-M with 80 links required only 40 seconds of computation time on a $2 \mathrm{GHz}$ Intel Core Duo machine with $2 \mathrm{~GB}$ of RAM. In most cases for which the optimal value is known, SMR-M produced somewhere between $10 \%$ and $15 \%$ lower throughput than optimal. So, while computing exact optimal solutions for large numbers of links is infeasible with a tool such as CPLEX, an existing heuristic MIMO scheduler modified to work in multihop networks can scale to quite large numbers of links and produces solutions that are not too bad.

To understand the limitations of Algorithm SMR-M, we looked at solutions produced for a $4 \times 4$ grid with type 2 link set and interference level 2. The link set has 8 links and the conflict graph contains two chains of 4 links each. SMR-M finds a solution where two streams are allocated to each link, which is the maximum number of streams that are feasible in this case. However, the optimal solution computed by CPLEX (see Figure $8^{4}$ ) allocates two streams to links on the ends of the chains but only one stream on the internal links. In this example, the optimal solution has some links transmitting fewer streams than possible, which reduces interference and allows the other links to use more DOFs for transmission or reception. Here, the array and diversity gains that result from the extra DOFs used in transmission/reception more than outweigh the reduced number of streams.

At an intermediate step of StreamMaxRate, the algorithm does not know exactly how all of the DOFs will end up being used, so it conservatively estimates that the minimum number of DOFs necessary to transmit a given number of streams will

\footnotetext{
${ }^{4}$ The numbers above the links indicate the numbers of streams allocated, the numbers inside the squares indicate the use of DOFs for interference suppression, and the numbers inside the circle indicate DOFs remaining for transmission or reception after DOFs used for suppression are subtracted.
} 


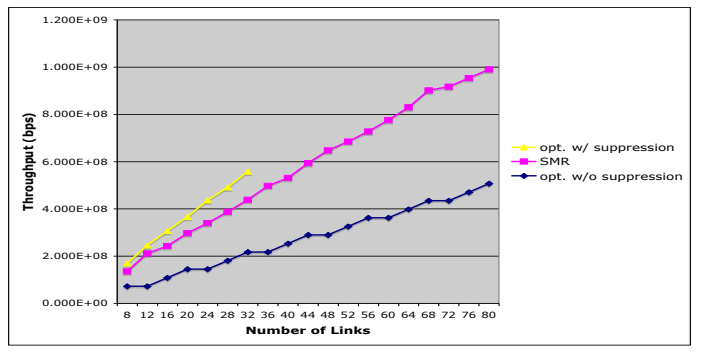

Fig. 9. Optimal throughput without interference suppression compared to throughputs obtainable with suppression (int. level $5, k_{i}^{t}=k_{i}^{r}=4, \forall i$ )

be used. Hence, diversity and array gains are not considered by StreamMaxRate during its intermediate steps. This tends to bias StreamMaxRate toward solutions that maximize the total number of streams in the network. Thus, StreamMaxRate does a good job of trading off spatial multiplexing and interference suppression, but does not adequately account for the additional factors of diversity and array gains. Development of heuristics that can account for the trade-offs among all of these different MIMO capabilities is an interesting open problem.

Comparison to No Interference Suppression Case:

It is interesting to see how much benefit is derived from using the interference suppression capabilities of MIMO. Here, we compare the solutions obtained so far to the optimal throughput without interference suppression. If interference suppression is not done, two links that are adjacent in the conflict graph cannot transmit concurrently, since there is no way to eliminate the interference between them. Hence, the optimal solution consists in finding a maximum independent set of the conflict graph and then using the DOFs on each of those links to maximize individual link rates. For the grid networks considered herein, it is simple to calculate this optimal value for a given interference level. The results are shown in Figure 9 for interference level 5. The result is compared to both the optimal value and the result from SMR$\mathrm{M}$ for type 2 link sets and the same interference level.

The results show that dramatic throughput increases are possible by adding interference suppression capability. The SMR-M heuristic is able to find solutions having approximately double the throughput of the best possible solution without interference suppression. The optimal possible with interference suppression is an additional 10-15\% higher. To take advantage of the potentially huge benefits of interference suppression shown in Figure 9, multiple-link optimization, such as we consider herein, is required.

\section{CONCLUSION}

This paper has demonstrated that exact optimal one-shot scheduling to maximize MIMO network throughput is possible for multihop wireless networks of reasonable size but heuristic schedulers that handle the diversity-multiplexing-interference suppression trade-off are needed for larger networks. Nearoptimal link scheduling, where demands on all links of a network must be met over multiple scheduling slots, is an open problem that is the subject of current research.

\section{ACKNOWLEDGEMENTS}

D. Blough was supported in part by Grants CNS-0721596 and CNS-1017248 from the National Science Foundation. G. Resta and P. Santi were supported in part by the Italian ccTLD Registration Authority under the Mimonet Project.

\section{REFERENCES}

[1] E. Baccarelli, et al., "Interference Suppression in MIMO Systems for Throughput Enhancement and Error Reduction," Proceedings of the International Conference on Wireless Communications and Mobile Computing, 2006.

[2] J. Barry, E. Lee, and D. Messerschmitt, Digital Communication, Springer, 2003.

[3] R. Bhatia, L. Li, "Throughput Optimization of Wireless Mesh Networks with MIMO Links", Proc. IEEE Infocom miniconference, pp. 2326-2330, 2007.

[4] G. Brar, D. Blough, and P. Santi, "Computationally Efficient Scheduling with the Physical Interference Model for Throughput Improvement in Wireless Mesh Networks," Proc. ACM MobiCom, pp. 2-13, 2006.

[5] S. Chu and X. Wang, "Adaptive and distributed scheduling in heterogeneous MIMO-based ad hoc networks," Proceedings of IEEE Int'l Conference on Mobile Ad Hoc Sensors and Systems (MASS), pp. 217-226, 2009.

[6] G. Foschini, M. Gans, "On Limits of Wireless Communications in a Fading Environment when Using Multiple Antennas", Wireless Personal Communications, Vol. 6, pp. 311-335, 1998.

[7] D. Gesbert, M. Shafi, D. Shiu, P. Smith, A. Naguib, "From Theory to Practice: an Overview of MIMO Space-Time Coded Wireless Systems", IEEE Journal on Selected Areas in Communications, Vol. 21, pp. 281-302, 2003.

[8] A. Goldsmith, European Wireless 2010, Keynote presentation.

[9] O. Goussevskaia, Y. Oswald, and R. Wattenhofer, "Complexity in Geometric SINR," Proc. ACM Mobihoc, pp. 100-109, 2007.

[10] B. Hamdaoui, K.G. Shin, "Characterization and Analysis of Multi-Hop Wireless MIMO Network Throughput", Proc. ACM MobiHoc, pp. 120-129, 2007.

[11] B. Hamdaoui, K. Shin, and M. Maiya, "Constraint Design and Throughput Evaluation in Multi-Band Wireless Networks using MIMO Links," IETE Technical Review, pp. 101-107, 2009.

[12] IEEE 80211n-2009-Amendment 5: Enhancements for Higher Throughput, IEEE Standards Association, Oct. 2009. (http://dx.doi.org/10.1109/IEEESTD.2009.5307322)

[13] J. Liu, Y.T. Hou, and H. Sherali, "Routing and Power Allocation for MIMO-based Ad Hoc Networks with Dirty Paper Coding," Proceedings of ICC, 2008.

[14] J. Liu, Y. Shi, and Y.T. Hou, "A Tractable and Accurate CrossLayer Model for Multi-hop MIMO Networks," Proceedings of Infocom, 2010.

[15] A.F. Molisch, Wireless Communications, John Wiley and Sons, Chichester, UK, 2005.

[16] B. Mumey, J. Tang, and T. Hahn, "Algorithmic Aspects of Communications in Multihop Wireless Networks with MIMO Links," Proceedings of ICC, 2010.

[17] R. Srinivasan, D. Blough, P. Santi, "Optimal One-Shot Stream Scheduling for MIMO Links in a Single Collision Domain", Proc. IEEE SECON, 2009.

[18] R. Srinivasan, D. Blough, L.M. Cortes-Peña, P. Santi, "Maximizing Throughput in MIMO Networks with Variable Stream Rates", Proc. European Wireless, 2010.

[19] K. Sundaresan, W. Wang, and S. Eidenbenz, "Algorithmic Aspects of Communication in Ad-hoc Networks with Smart Antennas," Proceedings of MobiHoc, 2007.

[20] J. Wang, et al., "Joint bandwidth allocation, element assignment and scheduling for wireless mesh networks with MIMO links," Computer Communications, Vol. 31, pp. 1372-1384, 2008. 